Ann. Sc. forest, 1978, 35 (1), 19-32.

\title{
Quelques résultats de cinétique journalière du potentiel de sève chez les arbres forestiers
}

\author{
G. AUSSENAC et A. GRANIER \\ Station de Sylviculture et de Production CNRF, Champenoux, 54280 Seichamps
}

\section{Résumé}

En 1976, année exceptionnellement sèche, les auteurs ont étudié dans le Nord-Est de la France, la cinétique journalière du potentiel de sève chez quelques arbres forestiers de 4 à $8 \mathrm{~m}$ de hauteur (Abies nordmanniana Spach, Cedrus atlantica Manetti, Cedrus deodora Loud., Cedrus libani (G. Don.) Loud., Picea abies (L) Karsten, Pinus nigra Arn. Ssp Nigricans Host., Pinus sylvestris L., Pseudotsuga menziesii (Mirb) Franco.

Certains arbres ont été soumis à la sécheresse estivale exceptionnellement grave, d'autres ont été arrosés.

L'analyse des cinétiques journalières du potentiel de sève a fait apparaître des différences entre les arbres bien alimentés en eau et ceux soumis à la sécheresse. Ces différences se sont marquées au niveau du potentiel de base $\left(P_{B}\right)$ et dans l'amplitude $(\Delta P)$ des oscillations journalières du potentiel de sève.

Chez les arbres arrosés, les valeurs du potentiel de base sont les suivantes : $-3,0$ à $-4,0$ bars chez Pseudotsuga menziesii, - 8,5 bars chez Abies nordmanniana, - 7,0 bars chez Pinus nigra, - 6,0 bars chez Pinus sylvestris, $-7,5$ bars chez Picea abies.

Au fur et à mesure que la sécheresse s'aggrave, $\Delta \mathrm{P}$ diminue et tend vers $0 ; \Delta \mathrm{P}$ reflète ainsi l'intensité de la fermeture stomatique. Les auteurs proposent alors une notion supplémentaire: le potentiel de base critique $\mathrm{P}_{\mathrm{B}_{0}}$ qui est défini comme le potentiel de base atteint lorsque l'amplitude des variations diurnes de potentiel est inférieure à 4 bars (pour une belle journée).

L'étude comparée du potentiel de sève chez Pseudotsuga menziesii, Pinus nigra et Pinus sylvestris permet de mettre en évidence des différences nettes de comportement de ces essences particulièrement entre le Douglas et les Pins. Les auteurs interprètent ces comportements de la façon suivante :

- Chez les pins, la transpiration et la photosynthèse sont élevées pour une alimentation en eau correcte. Lorsque la sécheresse s'installe, il y a diminution rapide à la fois de la transpiration ef de la photosynthèse avec un blocage de ces activités physiologiques pour des $P_{B_{0}}$ relativement forts (-16,0, - 17,0 bars). A partir de ce moment, la transpiration n'étant plus que cuticulaire, $P_{B}$ n'augmente que très lentement et dans ces conditions, l'arbre résiste bien à la sécheresse.

- Le Douglas continue à transpirer et à photosynthétiser jusqu'à des potentiels hydriques bas (- 21 bars). Ainsi, le blocage de ses activités intervient à des potentiels beaucoup plus faibles que ceux des pins. Ce potentiel hydrique faible risque cependant d'entraîner un état critique pour l'arbre lorsque la sécheresse s'aggrave.

\section{1. - Introduction}

Une façon de connaître l'état hydrique des arbres est de mesurer le potentiel de l'eau dans leurs organes, en particulier dans la partie feuillée. L'état de liaison de l'eau dans les feuilles, ou potentiel hydrique, est sous la dépendance de trois composantes : 
- deux facteurs externes qui sont les contraintes climatiques d'une part (énergie disponible au niveau des couronnes, pouvant être représentée par l'évapotranspiration potentielle), et la disponibilité en eau du sol d'autre part ;

- un facteur biologique interne, qui est la réponse de l'arbre qui réalise la jonction entre la demande en eau (atmosphère) et l'offre du sol. La variation des résistances au transfert et l'importance des réserves d'eau échangeable dans les organes permet à l'arbre de moduler sa réponse.

Une analogie électrique s'appuyant sur la loi d'Ohm, permet d'écrire :

$$
E T=\frac{\Delta \psi}{R}
$$

où ET représente la transpiration de la plante ou de l'arbre ; $\Delta \psi$ la différence de potentiel hydrique entre le sol ef l'atmosphère; et $R$ la résistance totale au transfert de l'eau entre le sol et l'atmosphère. Ce terme est d'ailleurs lui-même la somme de résistances intermédiaires, celle s'opposant au transit de l'eau depuis le sol jusqu'aux tissus conducteurs des racines, celle des tissus conducteurs jusqu'aux feuilles, et enfin celle qui existe entre les feuilles ef l'atmosphère. Cette analogie n'est valable qu'en régime de flux conservatif, c'est-à-dire lorsque la transpiration est égale à l'absorption.

Plusieurs auteurs ont mesuré le potentiel hydrique des tissus végétaux à l'aide de techniques difficiles à utiliser pour établir des cinétiques journalières. La méthode proposée par Scholander ef al. (1965) qui consiste à mesurer la pression de la sève dans les rameaux est d'une utilisation beaucoup plus facile. C'est celle que nous avons utilisée pour connaître (en conditions naturelles ou contrôlées) la réponse de différentes essences et la manière dont il était possible de caractériser leur état hydrique à un moment donné, ainsi que son évolution dans le temps.

\section{2. - Matériel et méthodes}

- L'étude a été menée en forêt domaniale d'Amance (Meurthe-et-Moselle), altitude $250 \mathrm{~m}$ et en forêt communale de Rouvres (Haute-Marne), altitude $400 \mathrm{~m}$.

Plusieurs espèces ont été étudiées suivant différentes modalités (†abl. 1). Les manipulations ont été réalisées dans le courant de l'été 1976, qui a été exceptionnellement chaud et sec. La figure 1 donne l'évolution théorique des réserves en eau pour des sols ayant des réserves utiles de $100 \mathrm{~mm}$ à Amance et de $50 \mathrm{~mm}$ à Rouvres. Cette réserve théorique a été calculée en tenant compte à la fois de l'ETP (évapotranspiration potentielle) calculée par la formule de Thornthwaite et des pluies pour un sol ayant une réserve utile donnée $=100 \mathrm{~mm}$ à Amance et $50 \mathrm{~mm}$ à Rouvres.

On écrit

$$
\mathrm{R}_{\mathrm{i}}=\mathrm{R}_{\mathrm{i}-1}-\mathrm{ETP}_{\mathrm{i}}+\mathrm{P}_{\mathrm{i}}
$$

$\mathrm{R}_{\mathrm{i}}=$ réserve hydrique du sol pour la période $\mathrm{i}$

$\mathrm{R}_{\mathrm{i}-\mathbf{1}}=$ idem pour la période $\mathrm{i}-1$

ETP $_{\mathrm{i}}=$ évapotranspiration potentielle pour la période $\mathrm{i}$ $P_{i}=$ total des pluies pour la période $i$.

Il faut remarquer que cette méthode sous-estime vraisemblablement la réserve réelle du sol à un moment donné, car d'une part, l'ETP représente une évapotrans- 
TABLEAU 1

Caractéristiques des espèces étudiées et modalités d'expérience

Description of studied species and treatments

$\begin{array}{lll}\text { Situation et espèces } & \begin{array}{c}\text { Age } \\ \text { (ans) }\end{array} & \begin{array}{c}\text { Hauteur } \\ (\mathrm{m})\end{array}\end{array} \quad$ Modalités et périodes d'étude

Forêt d'Amance

Cedrus atlantica Manetti provenance Algérie ...... Cedrus atlantica Manetti provenance Maroc ......

Cedrus deodara Loud.

provenance Inde .........

Cedrus libani (G. Don) Loudon

provenance Turquie......

Pseudotsuga menziesii

(Mirb.) Franco

provenance Shelton ......

provenance Winderhere

Lake

provenance Alberta ......

13

\section{Forêt de Rouvres}

Abies nordmanniana Spach
Picea abies (L.) Karsten...
Pinus nigra Arn. Ssp Nigri-
cans Host.
Pinus sylvestris L. . . . . . . . .

Pseudotsuga menziesii

(Mirb.) Franco ........

14
15
15
15

2,2

3,0

4,5

8,0 deux modalités :

4,6 - arbres soumis à la sécheresse de l'été 1976

3,2 - arbres irrigués tout au long de la saison de végétation (Aussenac, 1977) mesures le 13 et 24 août 1976

une seule modalité :

arbre soumis à la sécheresse de l'été 1976

3,8 mesures les 13 et 24 août 1976
5,0 - arbres soumis à la sécheresse mais arrosées deux fois les 15 et 16 juin, mesures du 15 au 20 juin 1976

piration qui n'est pas atteinte surtout en début de saison de végétation, el d'autre part parce que la disponibilité de la réserve n'est pas uniforme. La sécheresse s'est installée à Amance fin avril et s'est maintenue jusqu'au début du mois de septembre. A Rouvres, elle s'est manifestée de façon très nette à partir du 10 mai et a duré jusqu'au 20 septembre.

- Les mesures de potentiel ont été effectuées au moyen de la chambre à pression, technique développée depuis Scholander et al. (1965).

L'appareil utilisé a déjà été décrit par Aussenac et Chassagne (1974) et consiste en une chambre en acier inoxydable dans laquelle la partie feuillée du rameau est introduite, son extrémité basale dépassant par une ouverture du couvercle. De l'air comprimé est envoyé progressivement dans la chambre jusqu'à ce que la sève brute commence à sortir de la partie sectionnée. On relève à ce moment sur un manomètre la pression dans la chambre, que l'on appelle pression de sève.

Divers auteurs ont comparé les mesures de pression de sève et de potentiel hydrique des tissus, mesuré le plus souvent par la méthode psychrométrique. La relation 


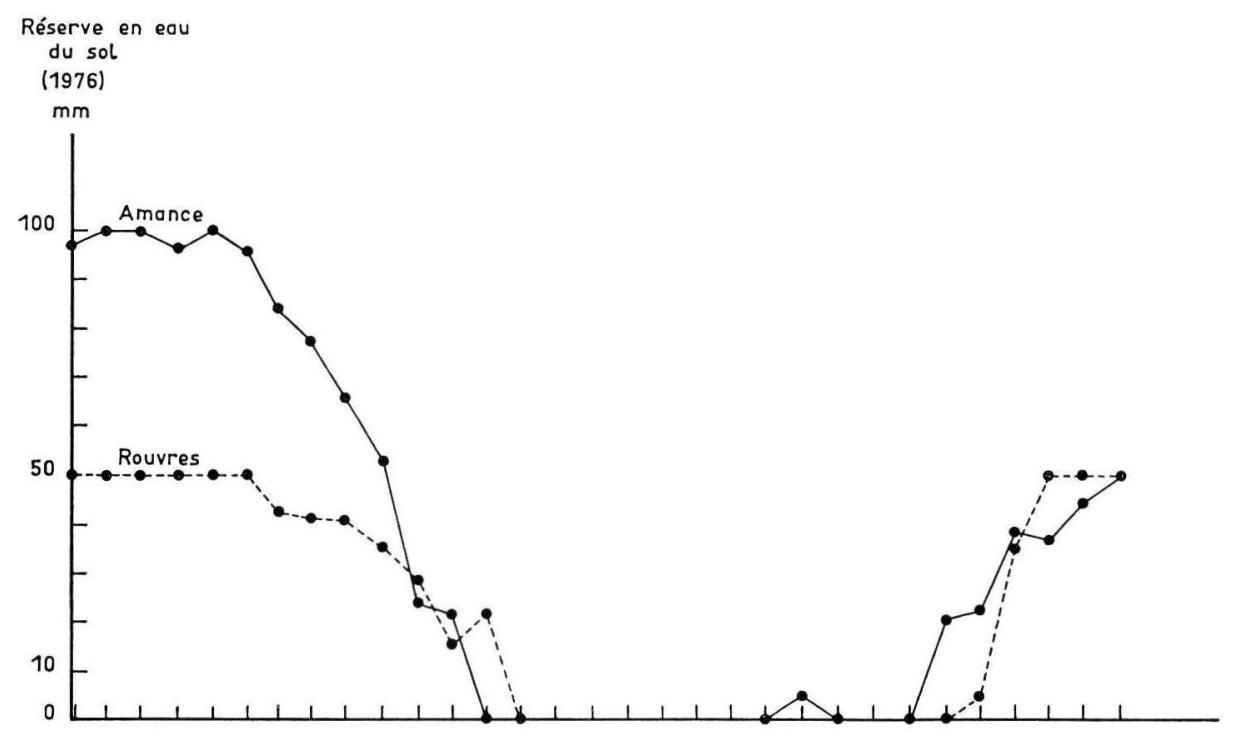

1102031102029102031102030102031102030102031102031102030102031 Dates

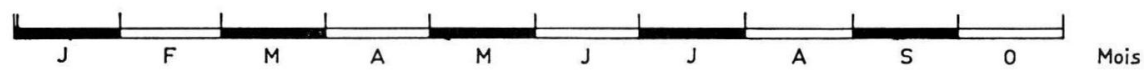

FIG. 1. - Evolution théorique de la réserve en eau du sol à Amance (-) et à Rouvres s/Aube (. . .) pour l'année 1976.

$$
\begin{aligned}
& \text { Réserve utile }= 100 \mathrm{~mm} \text { à Amance. } \\
& 50 \mathrm{~mm} \text { à Rouvres/s/Aube. }
\end{aligned}
$$

Theoretical evolution of soil water content in Amance (-) and Rouvres sur Aube (. . .) in 1976.

Available soil water : $100 \mathrm{~mm}$ in Amance.

$50 \mathrm{~mm}$ in Rouvres sur Aube.

entre ces deux grandeurs est dans la plupart des cas, linéaire, mais Kaufmann (1968) a montré que chez les feuillus, la relation pouvait être curviligne.

Le potentiel de sève a été exprimé ici en bars $\left({ }^{1}\right)$. Par convention, un potentiel a une valeur négative, ou nulle s'il s'agit de l'eau libre.

Les mesures ont été effectuées en utilisant des rameaux âgés de 1 an, prélevés toujours à la même hauteur (même verticille) et dans la même exposition (Sud).

\section{3. - Résultats}

3.1. - Influence des conditions hydriques sur la cinétique journalière du potentiel de sève

L'évolution journalière du potentiel de sève est sous la dépendance de l'humidité du sol d'une part et de l'évapotranspiration d'autre part. La cinétique est la suivante pour une belle journée : diminution du potentiel de sève (augmentation en valeur abso-

( ${ }^{1}$ ) Dans le système S. I. I'unité de potentiel est J. $\mathrm{m}^{-3}$ ou J. $\mathrm{kg}^{-1}$ selon qu'il est exprimé par unité de volume ou par unité de masse. 
lue) depuis le lever du soleil jusqu'à un minimum $\left(P_{m}\right)$ en milieu de journée et augmentation ensuite le soir jusqu'à la fin de la nuit : potentiel de base $P_{B}$. Hinckley et Ritchie (1973) ont défini le potentiel de base comme le potentiel d'équilibre entre l'arbre et le sol lorsque la transpiration est nulle ; il est atteint en fin de nuit.

L'évolution journalière des potentiels de sève a été étudiée chez deux Douglas (Pseudotsuga menziesii), l'un (témoin) poussant en conditions naturelles (sécheresse estivale prononcée), l'autre étant irrigué de manière à combler le déficit hydrique (fig. 2). A 5 h (T.U.), le potentiel de base est de - 4,5 bars pour l'arbre irrigué, alors qu'il atteint -16 bars dans le cas de l'arbre témoin. Dans la journée, la valeur minimale du potentiel qui est atteinte par les deux arbres est la même ( -21 bars). II en résulte donc que l'amplitude des oscillations est beaucoup plus grande chez l'arbre irrigué (16,5 bars) que chez l'arbre en conditions de sécheresse ( 5 bars). En serre, Granier (1975) observe le même phénomène sur deux jeunes Douglas âgés de huit ans, mais le potentiel minimum ( -19 bars) qui est atteint par les arbres arrosés est plus important que celui des arbres cultivés en conditions de sécheresse $(-25$ bars). L'amplitude des oscillations est dans ce cas de 3 bars pour l'arbre sec et de 13 bars pour l'arbre arrosé.

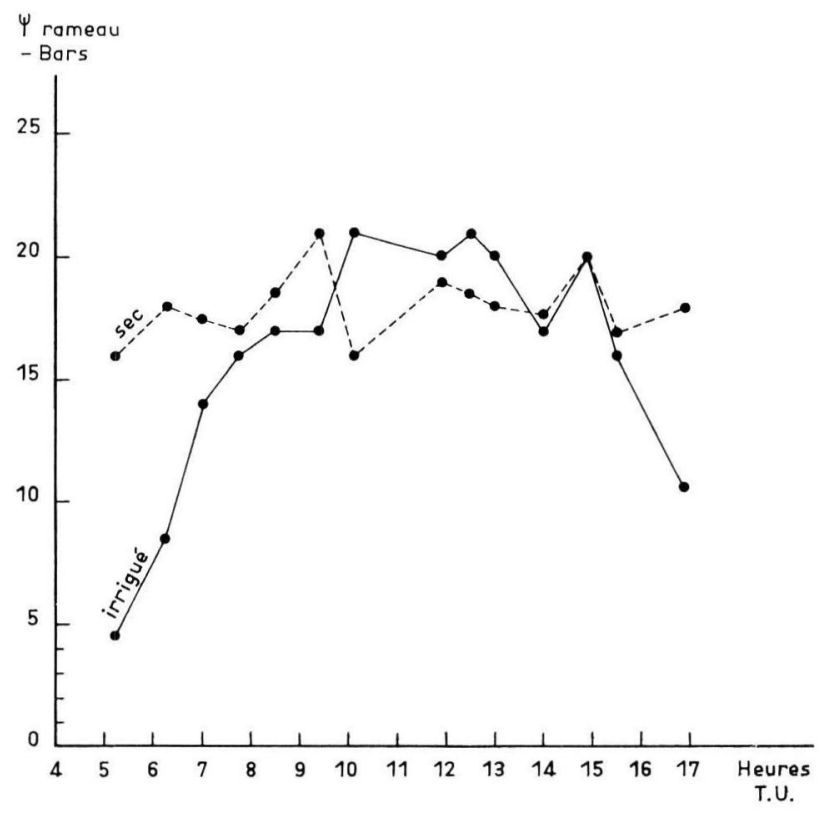

FIG. 2. - Evolution journalière du potentiel de sève chez Pseudotsuga menziesii (provenance Shelton) pour un arbre irrigué (-) et pour un arbre soumis à la sécheresse (- ....-), le 24.8.1976 à Amance.

Daily evolution of xylem sap pressure measured in Pseudotsuga menziesii (Shelton provenance) for an irrigated (-) and a non irrigated tree drought (-....) 24.08.1976 in Amance.

Les conditions de transpiration ont elles aussi une incidence sur la cinétique du potentiel de sève. Une baisse de transpiration s'accompagne d'une augmentation du potentiel. Sur la figure 3, on remarque le matin l'influence de la rosée : tant que les 

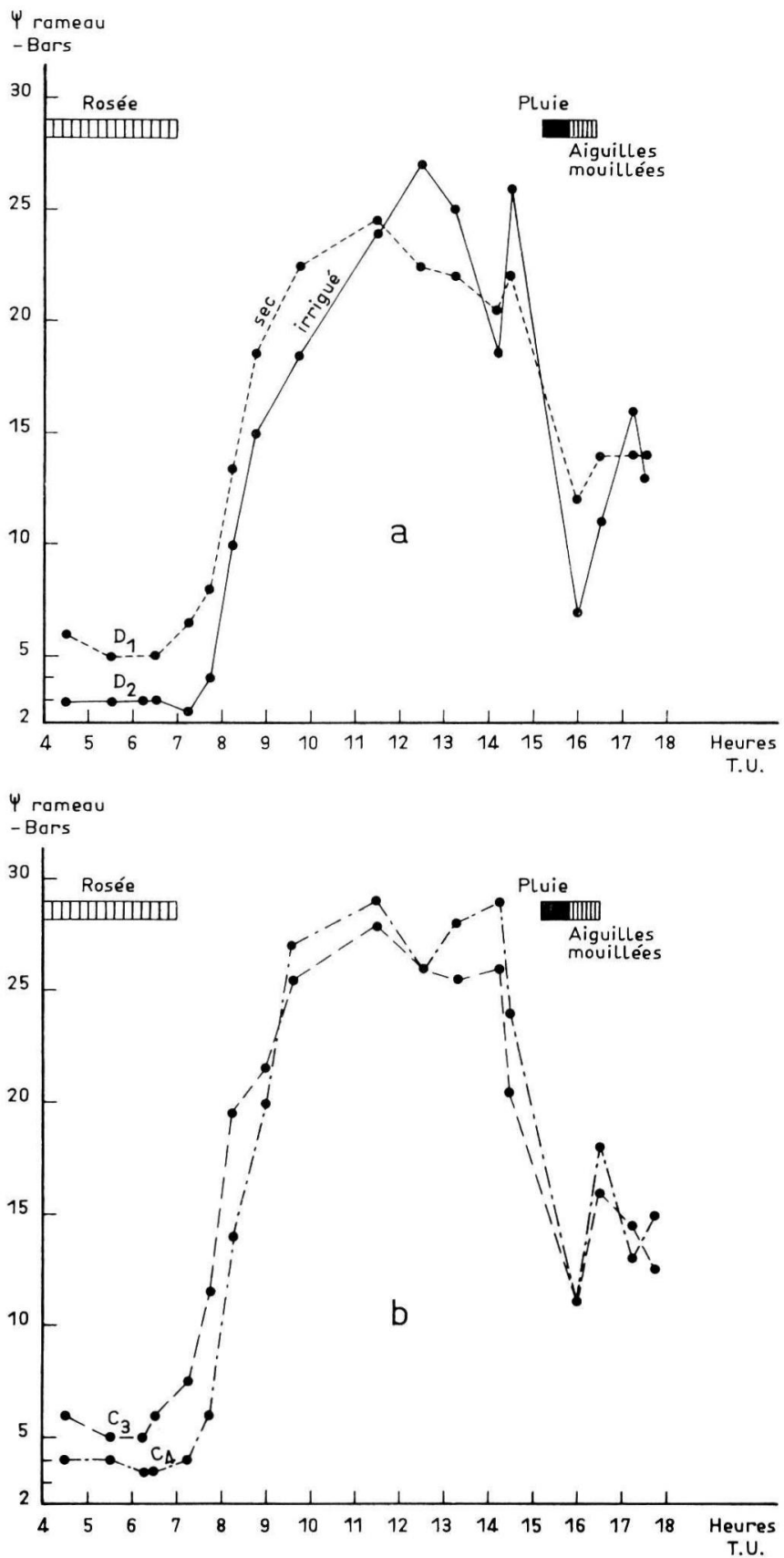

FIG. 3. - Evolution journalière du potentiel de sève, le 13.8.1976 à Amance. a) Pseudotsuga menziesii irrigué (-) ef sec (-..-) b) Cedrus atlantica : deux provenances $C_{3}$ (Maroc) et $C_{4}$ (Algérie) (arbres soumis à la sécheresse).

Daily evolution of xylem sap pressure in Amance (13.08.76). a) Pseudotsuga menziesii irrigated (-) and non irrigated tree (-...-.). b) Cedrus atlantica two origins: $C_{3}$ (Morroco) and $C_{1}$ (Algeria). These trees were non watered. 
aiguilles sont mouillées, le potentiel reste élevé aussi bien pour l'arbre témoin (sec) que l'arbre irrigué. Le potentiel diminue ensuite rapidement (à partir de $7 \mathrm{~h} 30$ ). Le soir, une pluie très légère $(0,1 \mathrm{~mm})$ suffit à le faire augmenter notablement, surtout chez l'arbre irrigué. Dès que l'eau interceptée par le feuillage est évaporée, le potentiel diminue à nouveau. La baisse de la transpiration due à l'évaporation de l'eau interceptée (rosée ou pluie) a pour conséquence une recharge de l'arbre à partir de l'eau du sol. Compte tenu de la brièveté de la séquence pluvieuse, il est probable que l'augmentation du potentiel qui a été mesurée correspond à une faible recharge en eau.

\section{2. - Etude comparative des cinétiques journalières du potentiel de sève chez quelques essences forestières}

La figure 4 montre l'évolution des potentiels de sève au cours de journées successives chez Abies nordmanniana, Picea abies, Pinus nigra et Pinus sylvestris. Chez les pins (fig. 4a), les témoins (arbres poussant en conditions naturelles : sécheresse prononcée) ont des potentiels de sève qui varient très peu au cours de la journée (2 bars). Ils se situent aux environs de -15 bars pour les pins sylvestres et -17 bars pour les pins noirs. Ces valeurs représentent les potentiels de base $\left(P_{B}\right)$ pour ces espèces, dans les conditions de la station. Les arbres qui ont été arrosés présentent au cours du deuxième jour une augmentation générale du potentiel. Le potentiel de base est alors de -6 bars pour le pin sylvestre et de -7 bars pour le pin noir. Dans la journée, la fluctuation du potentiel a légèrement augmenté. Dans le courant du troisième jour, les courbes des arbres arrosés et des arbres témoins se rapprochent. Pour ces trois journées, on remarque que les pins noirs et les pins sylvestres ont un comportement identique. Chez l'épicéa (fig. $4 b$ ), les témoins ont un potentiel de base plus bas que celui des pins ( -19 bars), mais il présente également une fluctuation faible dans le courant de la journée (4 bars). Chez les arbres arrosés, on note une augmentation importante de potentiel de base ( $-7,5$ bars) et une fluctuation journalière plus accusée (6 bars) le premier et le deuxième jour après l'arrosage. Les courbes des arbres arrosés et des arbres secs sont très décalées comme c'était le cas chez le pin.

Chez le sapin de Nordmann (fig. 4c), les potentiels de l'arbre arrosé sont voisins de ceux du témoin dans la journée. Toutefois, les potentiels de base sont nettement différents : $-8,5$ bars pour l'arbre arrosé et -12 bars pour l'arbre sec. Le tableau 2 donne pour les arbres étudiés la valeur du potentiel de base et du potentiel minimum $P_{m}$ mesurés à Rouvres et à Amance.

Sur ce tableau, sont également reportées les valeurs d'un paramètre supplémentaire : le potentiel de base critique $P_{B_{0}}$ que nous définissons comme le potentiel de base atteint lorsque l'amplitude des variations diurnes de potentiel est inférieure à 4 bars (pour une belle journée). C'est le cas de Picea abies, Pinus nigra et Pinus sylvestris à Rouvres.

\section{4. - Discussion et conclusion}

L'analyse des cinétiques journalières du potentiel de sève chezPseudotsuga menziesii a fait apparaître des différences entre les arbres bien alimentés en eau et ceux soumis à la sécheresse (fig. 2). Ces différences se manifestent au niveau du potentiel 


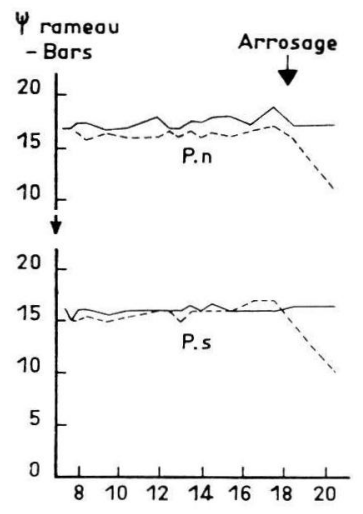

$15 / 6$

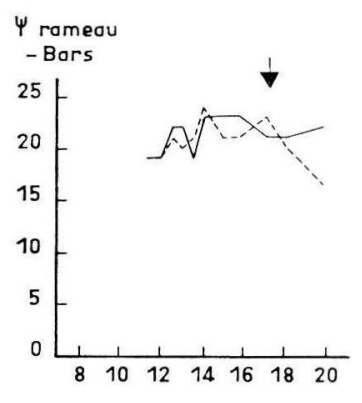

$15 / 6$
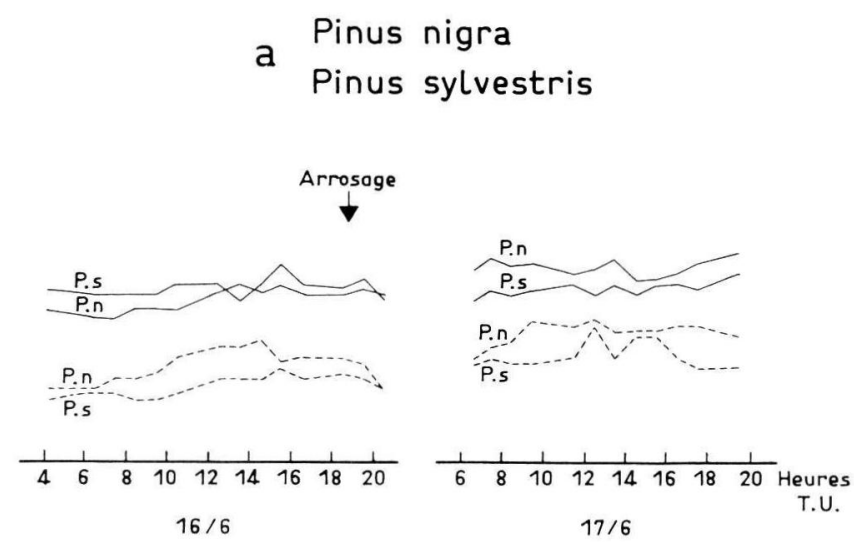

b Picea abies

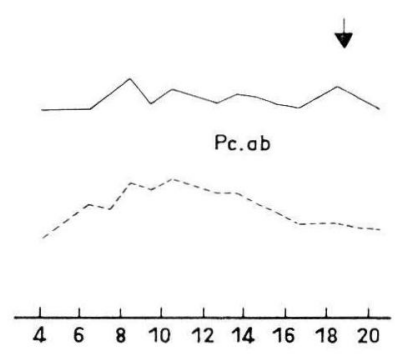

$16 / 6$

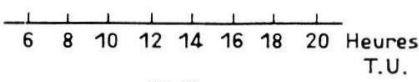

$17 / 6$

\section{Abies nordmanniana}

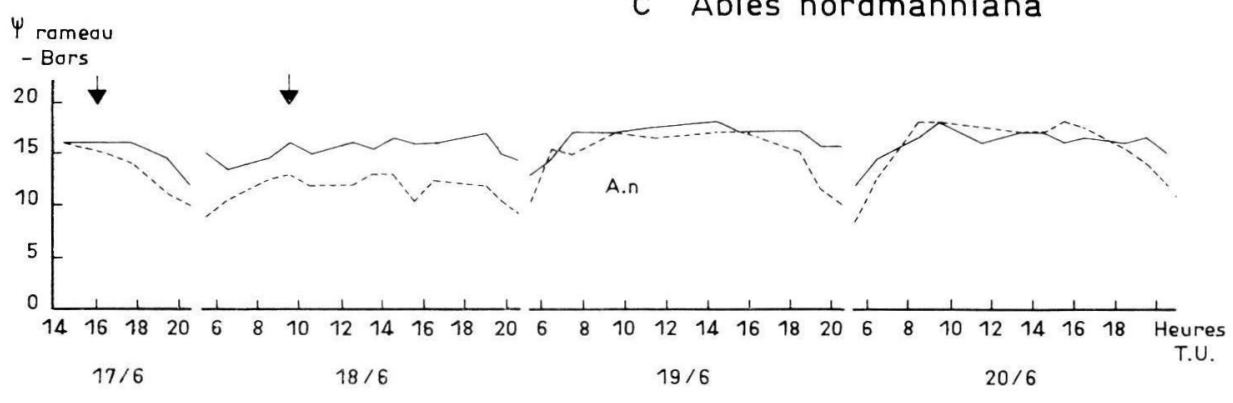

1976

FIG. 4. - Evolution journalière du potentiel de sève lors de journées successives à Rouvres (1976) pour des arbres secs (-) et pour des arbres arrosés (-...-). a) Pinus nigra et Pinus sylvestris. b) Picea abies. c) Abies nordmanniana.

Daily evolution of xylem sap pressure during successive days in Rouvres (1976) for droughty (-) and irrigated trees (-...-.). a) Pinus nigra and Pinus sylvestris. b) Picea abies. c) Abies nordmanniana. 


\section{TABLEAU 2}

Potentiel de base, potentiel minimum et potentiel de base critique des espèces étudiées Basic potential, minimum potential, critical basic potential for the species studied

\begin{tabular}{|c|c|c|c|c|c|}
\hline \multirow{2}{*}{ Espèces } & \multicolumn{2}{|c|}{$\begin{array}{c}\text { Potentiel } \\
\text { de base }\left(P_{B}\right)\end{array}$} & \multicolumn{2}{|c|}{$\begin{array}{l}\text { Potentiel minimum } \\
\left(\mathrm{P}_{\mathrm{m}}\right)\end{array}$} & \multirow[t]{2}{*}{$\begin{array}{c}\text { P otentiel } \\
\text { de base critique } \\
\left(P_{\mathrm{B}_{0}}\right)\end{array}$} \\
\hline & $\begin{array}{l}\text { Arbres } \\
\text { secs }\end{array}$ & $\begin{array}{l}\text { Arbres } \\
\text { irrigués }\end{array}$ & $\begin{array}{l}\text { Arbres } \\
\text { secs }\end{array}$ & $\begin{array}{l}\text { Arbres } \\
\text { irrigués }\end{array}$ & \\
\hline \multicolumn{6}{|l|}{ Forêt d'Amance } \\
\hline $\begin{array}{l}\text { Cedrus atlantica (Algérie). } \\
\text { Cedrus atlantica (Maroc) } \\
\text { Cedrus deodara (Inde) ..... } \\
\text { Cedrus libani (Turquie) .. } \\
\text { Pseudotsuga menziesii } \\
\text { provenance Shelton ...... } \\
\text { provenance Winderhere } \\
\text { Lake ................. } \\
\text { provenance Alberta ...... }\end{array}$ & $\begin{array}{l}-13,0 \\
-12,0 \\
-14,0 \\
-14,0 \\
-16,0 \\
-14,0 \\
-10,0\end{array}$ & $\begin{array}{l}-4,5 \\
-\quad 3,0 \\
-\quad 4,0\end{array}$ & $\begin{array}{l}-28,0 \\
-28,5 \\
-25,0 \\
-26,0 \\
-21,0 \\
-20,0 \\
-24,0\end{array}$ & $\begin{array}{l}-21,0 \\
-18,0 \\
-25,0\end{array}$ & $-21,0$ \\
\hline \multicolumn{6}{|l|}{ Forêt de Rouvres } \\
\hline $\begin{array}{l}\text { Abies nordmanniana } \ldots \ldots \\
\text { Pinus nigra } \ldots \ldots \ldots \ldots \\
\text { Pinus sylvestris . . . . . . . . } \\
\text { Picea abies . . . . . . . } \\
\text { Pseudotsuga menziesii . . }\end{array}$ & $\begin{array}{l}-12,0 \\
-17,0 \\
-16,0 \\
-20,0 \\
-10,0\end{array}$ & $\begin{array}{r}8,5 \\
-\quad 7,0 \\
-\quad 6,0 \\
-\quad 7,5 \\
-10,0\end{array}$ & $\begin{array}{l}-18,0 \\
-20,0 \\
-19,9 \\
-24,0 \\
-20,0\end{array}$ & $\begin{array}{r}18,0 \\
-11,5 \\
-\quad 9,0 \\
-13,5 \\
-23,5\end{array}$ & $\begin{array}{l}17,0 \\
-16,0 \\
-20,0\end{array}$ \\
\hline
\end{tabular}

de base $P_{B}$, et dans l'amplitude $\Delta P$ des oscillations journalières du potentiel de sève $\left(\Delta \mathrm{P}=\mathrm{P}_{\mathrm{B}}-\mathrm{P}_{\mathrm{m}}\right)$.

Les valeurs du potentiel de base pour un sol bien alimenté en eau sont de l'ordre de -2 bars à -4 bars. Ces valeurs sont voisines de celles trouvées par d'autres auteurs : Pierpoint (1967) chez Picea glauca (- 4,2 bars), Larix laricina (- 4,9 bars), Hinckley et Ritchie (1973) chez Abies procera ( -2 bars), Abies amabilis ( -2 bars), Pinus contorta ( -1 bar) ; Waggoner et Turner (1971) chez Pinus resinosa ( -2 bars).

Les valeurs des potentiels de base chez les arbres soumis à la sécheresse sont beaucoup plus faibles : -16 bars (tabl. 2). La valeur atteinte dépend du niveau de sécheresse du sol : Sucoff (1972) a montré chez Pinus resinosa qu'il existait une relation étroite entre la teneur en eau du sol et le potentiel de base. On peut alors penser avec d'autres auteurs que $\mathrm{P}_{\mathrm{B}}$ permet d'évaluer la disponibilité en eau du sol à un moment déterminé.

La valeur de $\mathrm{P}_{\mathrm{m}}$ dépend à la fois de l'évapotranspiration potentielle et de la réponse propre à l'arbre (résistances au transfert de l'eau, avec en particulier la régulation stomatique).

Dans la figure 2 , nous avons vu que les valeurs de $P_{m}$ des arbres soumis à la sécheresse et des arbres irrigués étaient voisines ( -21 bars); il en résulte que la différence $\Delta \mathrm{P}=\mathrm{P}_{\mathrm{B}}-\mathrm{P}_{\mathrm{m}}$ est plus importante pour les arbres arrosés que pour les secs. Au fur et à mesure que le dessèchement du sol augmente et que la transpiration 
de l'arbre diminue, $\Delta \mathrm{P}$ baisse ef tend vers 0 . Ainsi, la valeur de $\Delta \mathrm{P}$ reflèterait l'intensité de la fermeture stomatique.

La dynamique du potentiel de sève en fonction de l'augmentation du potentiel hydrique du sol est schématisée sur la figure 5. Ce schéma représente les enveloppes supérieures $\left(P_{m}\right)$ et inférieures $\left(P_{B}\right)$ des variations journalières du potentiel de sève. La valeur de $P_{B}$ pour un $\Delta P$ faible correspond dans notre schéma à l'état hydrique atteint par l'arbre lorsque la fermeture des stomates est totale (transpiration extrêmement réduite), c'est ce que nous avons appelé $\mathrm{P}_{\mathrm{B}_{0}}$. Dans nos expérimentations, nous avons trouvé des valeurs de $\mathrm{P}_{\mathrm{B}_{0}}$ égales à -20 bars pour Picea abies, -21 bars pour Pseudotsuga menziesii, -17 bars pour Pinus nigra et -16 bars pour Pinus sylvestris. Ces valeurs sont voisines de celles qu'a mesuré Lopushinsky (1969) lorsque la fermeture stomatique est totale : $-17,3$ pour Pinus ponderosa, $-16,2$ bars pour Pinus contorta, $-22,5$ bars pour Pseudotsuga menziesii. Notre schéma est voisin de celui donné parWaring et Cleary (1967). On a l'habitude de définir l'état de sécheresse extrême d'un végétal au moyen du point de flétrissement temporaire et du point de flétrissement permanent, mais ces paramètres sont dans la pratique difficiles à évaluer. De plus Becker (1970) note que des jeunes plants résineux peuvent résister à des $p F$ supérieurs à 4,2 (point de flétrissement permanent). La valeur de $\mathrm{P}_{\mathrm{B}_{0}}$ pourrait alors être utilisée pour caractériser chez une espèce le moment où les processus de transpiration et de photosynthèse sont bloqués.

Dans le cas de Pseudotsuga menziesii, l'enveloppe supérieure $\left(P_{m}\right)$ dépasse $P_{B_{0}}$ quand le sol est peu desséché. Les études portant sur les autres essences : Pinus sylvestris, Pinus nigra, Picea abies permettent de tracer un schéma différent pour l'évolution de $P_{m}$ et de $P_{B}$ en fonction du potentiel hydrique du sol (fig. $5 b$ ). Dans ce cas, l'enveloppe du potentiel minima est inférieure à $\mathrm{P}_{\mathrm{B}_{0}}$. Pour des plantes arbustives et herbacées, Hickman (1970) trouve lui aussi différents types d'évolution de $P_{B}$ et de $P_{m}$ en fonction du dessèchement du sol. Dans l'expérimentation de Rouvres, on a remarqué que pour les mêmes journées, après une longue période de sécheresse, les pins et l'épicéa avaient un comportement différent du Douglas et du sapin de Nordmann. Les premiers avaient atteint ou dépassé $\mathrm{P}_{\mathrm{B}_{0}}$, alors que les autres se situaient encore au-dessus. La figure 6 schématise pour les pins et le Douglas l'évolution dans le temps des potentiels de sève, en période de dessèchement. On constate qu'au temps $t_{n}$, le Douglas a un $\mathrm{P}_{\mathrm{B}}$ supérieur à celui du pin, ce qui signifie que le sol prospecté par les racines de la première espèce est moins desséché que celui occupé par la deuxième. Les conditions évapotranspiratoires étant les mêmes, il en résulte que la transpiration des pins a été plus forte que celle du Douglas. C'est aussi ce qu'ont trouvé Lopushinsky et Klock (1974) qui ont montré que la transpiration des pins, lors d'une période de dessèchement, était très élevée les premiers jours, puis chutait brusquement. Chez Abies grandis et Pseudotsuga menziesii, la transpiration est beaucoup plus faible au départ, mais diminue très progressivement dans le temps. $P_{\mathrm{B}_{0}}(-25,8$ bars pour Abies grandis et $-22,5$ bars pour Pseudotsuga menziesii) est atteint beaucoup plus tardivement, au bout de 13 jours contre 6 jours pour Pinus resinosa.

Pour mieux analyser le comportement des essences forestières face à la sécheresse, il faut distinguer deux notions :

- la résistance à la sécheresse qui est la possibilité qu'a une espèce de subsister malgré le déficit hydrique, 


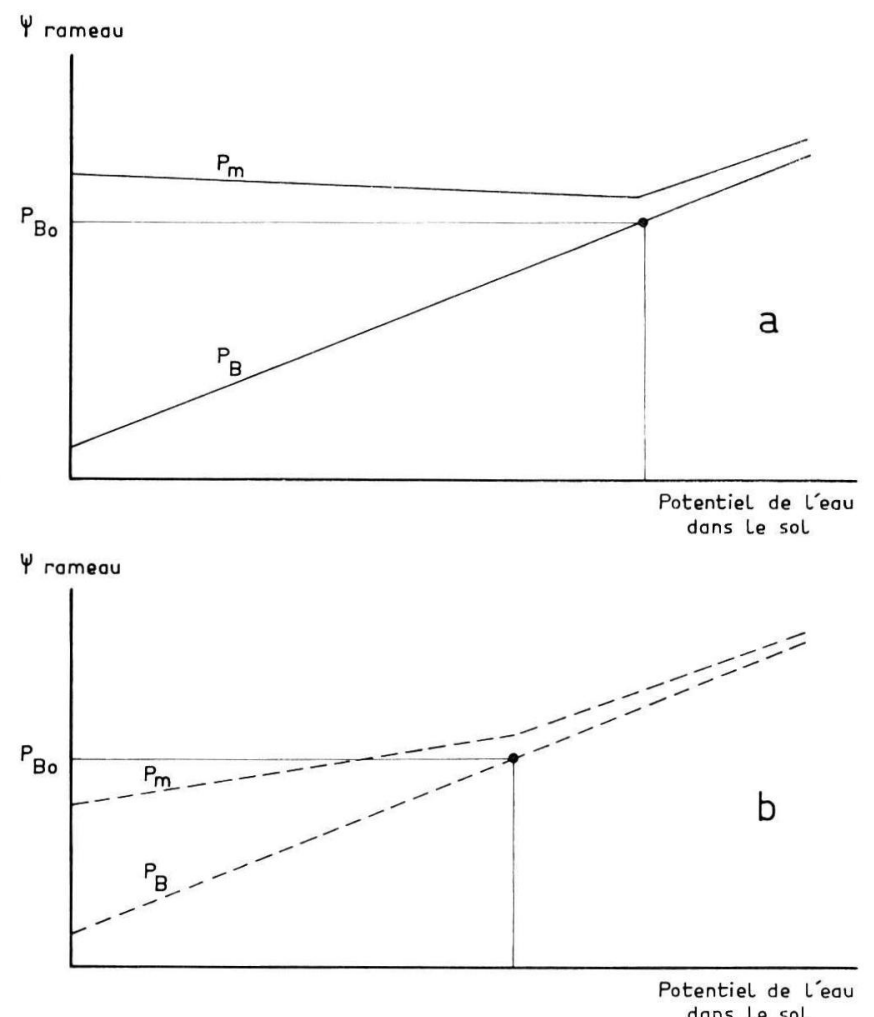

FIG. 5. - Schéma de l'évolution du potentiel de sève en fonction du dessèchement du sol. $P_{m}$ représente le potentiel minimum théorique et $P_{\mathrm{B}}$ le potentiel de base. a) Pseudotsuga menziesii. b) Pinus nigra.

Scheme of the evolution of xylem sap pressure versus drying soil. $P_{m}$ is the theoretical minimum potential and $P_{\mathrm{B}}$ the basic potential a) Pseudotsuga menziesii. b) Pinus nigra.

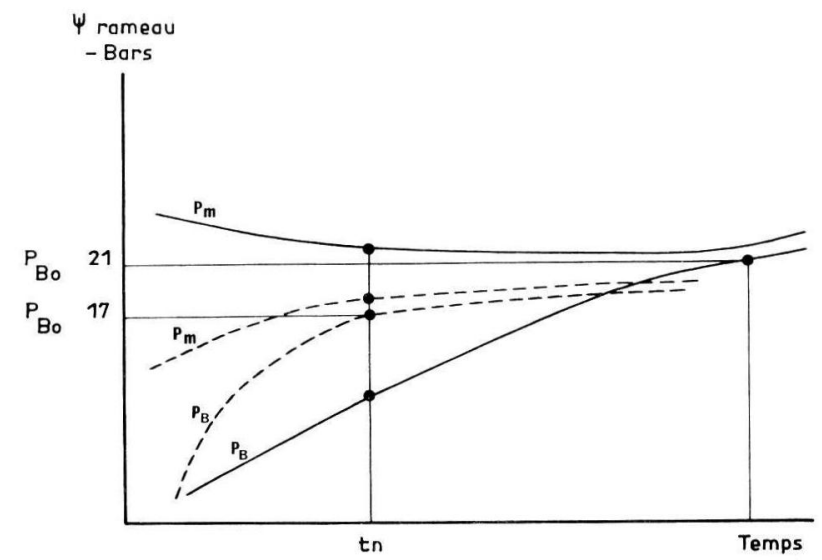

FIG. 6. - Schéma de l'évolution du potentiel de sève en fonction du temps. La figure représente les enveloppes supérieure $\left(P_{\mathrm{m}}\right)$ ef inférieure $\left(P_{\mathrm{B}}\right)$ pour deux espèces: Pseudotsuga menziesii $(-)$ ef Pinus nigra $(\longrightarrow)$.

Scheme of the evolution of xylem sap pressure versus time. This figure gives upper $\left(P_{\mathrm{m}}\right)$ and lower $\left(P_{\mathrm{B}}\right)$ envelopes for two species: Pseudotsuga menziesii (-) and Pinus nigra ( --$)$. 
- la possibilité pour l'arbre de continuer ses activités biologiques (photosynthèse, croissance) même pendant une période de déficit hydrique.

II semble que le comportement des pins (ceux que nous avons étudiés) puisse être interprété de la façon suivante : transpiration et photosynthèse importantes pour une alimentation en eau correcte, ensuite lorsque la sécheresse s'installe diminution rapide à la fois de la transpiration et de la photosynthèse avec un blocage de ces activités biologiques pour des $\mathrm{P}_{\mathrm{B}}$ relativement forts. A partir de ce moment, la transpiration n'étant plus que cuticulaire, le $P_{B}$ n'augmente que très lentement et dans ces conditions, l'arbre résiste bien à la sécheresse.

Le Douglas est capable de transpirer et de photosynthétiser à des potentiels hydriques plus faibles, ce qui lui donne, lors de sécheresses modérées (déficit pluviométrique peu important ou réserve en eau du sol suffisante) de grandes possibilités d'assimilation. Lorsque la sécheresse se prolonge, le meilleur comportement des pins, tel que les forestiers ont pu l'observer, peut sans doute s'expliquer par le maintien de leur état hydrique à un niveau plus satisfaisant que celui des autres résineux : $P_{B}$ (pins) $>P_{B}$ (Douglas) par exemple.

Reçu pour publication en juillet 1977.

\section{Summary}

Some results about daily kinetics of xylem sap pressure for forest trees

In the North Eastern part of France during 1976 which was an exceptionnaly dry year daily kinetics of xylem sap pressure for some forest trees ( 4 to $8 \mathrm{~m}$ height) were examinated (Abies nordmanniana Spach. Cedrus atlantica Manetti, Cedrus deodara Loud., Cedrus libani (G. Don) Loud., Picea abies (L) Karsten, Pinus nigra Arn. Ssp Nigricans Host., Pinus sylvestris L. Pseudotsuga menziesii (Mirb.) Franco). Some of the trees were left non irrigated during this period of exceptionnaly severe drought, other were watered.

Analysis of daily kinetics of xylem sap pressure have shown discrepancies between well-watered trees and droughty trees. These differences have been noticed on the basic potential $\left(P_{B}\right)$ and on the amplitude $(\Delta P)$ of the daily sap pressure variations.

For watered trees the following values of basic potential have been found : -3.0 to 4.0 bars for a Pseudotsuga menziesii, $-7,0$ bars for Pinus nigra, $-8,5$ bars for Abies nordmanniana, $-6,0$ bars for Pinus sylvestris, $-7,5$ bars for Picea abies. In increasing drought, $\Delta P$ decreases to 0 . $\Delta P$ is there reflecting the stomatal closure.

An additionnal concept is then offered by the authors. The critical basic potential $\mathrm{P}_{\mathrm{B}_{0}}$, defined as the basic potential reached when the amplitude of daily variations is less than 4 bars (during a sunny day). Comparative study of xylem sap pressure for Pseudotsuga menziesii, Pinus nigra and Pinus sylvestris displays marked differences of behaviour between these species, especially between Douglas-fir and pines. These behaviours are interpreted as follow :

- Pines have important transpiration and photosynthesis when enough water is available. In increasing the drought there is a rapid decrease in transpiration and photosynthesis; these physiological activities are stopped for relatively high $\mathrm{P}_{\mathrm{B}_{0}}$ ( -16 to -17 bars). From that moment the transpiration being only cuticular, $\mathrm{P}_{\mathrm{B}}$ increases only very slowly and the tree supports well the drought.

- Douglas-fir continues to have transpiration and photosynthesis till low water potential are reached ( -21 bars). There the blockage of its activities happens at very much lower potentials than pines. If the drought increases such a low water potential may induce a critical condition for the trees. 


\title{
Zusammenfassung
}

\author{
Einige Ergebnisse über den Tagesgang des Saftstrompotenzials in Koniferen
}

\begin{abstract}
Die Autoren untersuchten im aussergewöhnlich trocken Sommer 1976 denTagesgang des Saftstrompotenzials verschiedener Nadelhölzer (Abies nordmanniana Spach, Cedrus atlantica Manetti, Cedrus deodara Loud., Cedrus libani (G. Don.) Loud., Picea abies (L) Karsten, Pinus nigra Arn. Ssp Nigricans Host., Pinus sylvestris L., Pseudotsuga menziesii (Mirb/Franco)).

Die Untersuchungen wurden in Nordostfrankreich (Nancy und Langres) an 4-8 m hohen Bäumen durchgeführt. Ein Teil der Bäume wurde regelmänig bewässert, während die restlichen Bäume der extremen Sommertrockenperiode ausgesetzt blieben.

Die Analyse des Tagesganges des Saftstrompotenzials zeigt deutliche Unterschiede zwischen den bewässerten und den unbewässerten Bäumen, sowohl bezüglich des Basispotenzials $\left(P_{B}\right)$ als auch der Amplitude $(\Delta \mathrm{P})$ des Tagesganges.

Bei den bewässerten Bäumen wurden die folgenden Basispotenziale festgestellt : Pseudotsuga menziesii : - 3,0 bis - 4,0 Bar Abies nordmanniana :-8,5 Bar Pinus nigra : - 7,0 Bar, Pinus sylvestris : $-6,0$ Bar, Picea abies : $-7,5$ Bar.

Mit zunehmender Trockenheit nimmt die Amplitude des Tagesganges $(\Delta P)$ ständing ab und verschwindet gänzlich; die $\Delta P$-Werte bringen daher die Intensität des Stomataschlusses deutlich zum Ausdruck. Die Autoren schlagen daher den Begriff eines «kritischen Basispotenzials» $\left(P_{B_{0}}\right)$ vor, den sie als jenes Basispotenzial definieren das an einem Strodlungstag erreicht wird, wenn die Amplitude des Tagesganges $(\Delta P)$ 4,0 Bar beträgt.

Die vergleichende Untersuchung des Saftstrompotenzials bei Pseudotsuga menziesii, Pinus nigra und Pinus sylvestris zeigten deutliche Unterschiede insbesondere zwischen der Douglasie und den Kiefern. Dieses unterschiedliche Verhalten wird von den Autoren wie folgt interpretiert :

- Bei guter Wasserversorgung haben die Kiefern hohe Transpiration und Photosynthese, welche mit zunehmender Trockenheit rasch abnehmen und zu einer Blockierung der physiologischen Aktivität bei verhältnismässig hohen « kritischen Basispotenzialen » $\left(\mathrm{P}_{\mathrm{B}_{0}}\right)$ von $-16,0$ bis $-17,0 \mathrm{Bar}$ führen. Von diesem Zeitpunkt an ist die Transpiration nur mehr cuticulär und das Basispotenzial $\left(P_{B}\right)$ erhöht sich nur sehr langsam. Damst ergibt sich eine gute Widerstandsfähigkeit bei Wassermangel.

- Bei der Douglasie gehen Transpiration und Photosynthese bis zu einem sehr niederen Saftstrompotenzial (- 21,0 Bars) weiter. Die Blockierung der biologischen Aktivität erfolgt daher bei der Douglasie bei wesentlich geringeren Saftstrompotenzialen als bei den Kiefern. Bei weiter zunehmender Trockenheit kann dies zu irreversiblen Trockenschäden führen.
\end{abstract}

\section{Références bibliographiques}

AUSSENAC G., CHASSAGNE L., 1974. Rapport préliminaire sur la mesure du potentiel de la sève dans le rameau et les fevilles. Publication interne Station de Sylviculture et de production, 8 p.

AUSSENAC G., 1977. Premiers résultats de l'étude de l'influence de l'alimentation en eau sur la croissance des arbres dans un peuplement de Pseudotsuga menziesii (Mirb.) Franco. Publication interne Station de Sylviculture et de Production, $10 \mathrm{p}$.

BECKER M., 1970. Transpiration et comportement vis-à- vis de la sécheresse de jeunes plants forestiers Ann. Sci. For., 27 (4), 401-420.

GRANIER A., 1975. Transpiration et vitesse de sève de jeunes Douglas en relation avec leur état hydrique. Publication interne Station de Sylviculture et de Production, 25 p.

HICKMAN J. C., 1970. Seasonal course of xylem sap tension, Ecology, 51, 1052-1056.

HINCKLEY T. M., RITCHIE G. A., 1973. A theoretical model for calculation of xylem sap pressure from climatological datas. The American Midland Naturalist, 90 (1) 56-69.

KAUFMANN M. R., 1968. Evaluation of the pressure chamber technique for estimating plant water potential of forest trees species. Forest Science 14 (1) 369-374.

LOPUSHINSKY W., 1969. Stomatal closure in conifer seedlings in response to leaf moisture stress. Bot Gaz., 130 (4) 258-263.

LOPUSHINSKY W., KLOCK G. O., 1974. Transpiration of conifer seedlings in relation to soil water potential. Forest Science, 20 (2) 181-186.

PIERPOINT G., 1967. Direct measurement on internal moisture deficit in trees. Forest Chronicle, 43 , 145-148.

RITCHIE G. A., HINCKLEY T. M., 1975. The pressure chamber as an instrument for ecological research. Advances in Ecological research (A. Mac Fayden Ed.), vol. 9, 165-254. Academic Press London. 
SCHOLANDER P. F., HAMMEL H. T., BRADSTREET E. D., HEMMINGSEN E. A., 1965. Sap pressure in vascular plants. Science, 148, 339-246.

SUCOFF E., 1972. Water potential in red pine : soil moisture, evapotranspiration, crown position. Ecology, 53 (4) 681-686.

WAGGONER P. E., TURNER N. C., 1971. Transpiration and its control by stomata in a pine forest. Bulletin of the Connecticut Agricultural Experiment Station New Haven, 726, 87 p.

WARING R. H., CLEARY B. D., 1967. Plant moisture stress : evaluation by pressure bomb. Science, $155,1248-1254$. 